Pacific Journal of Mathematic 


\title{
LINKS WHICH ARE UNKNOTTABLE BY MAPS
}

\author{
HOWARD LAMBERT
}

\begin{abstract}
Let $L$ be a piecewise linear (PL) link of two components in the Euclidean 3-sphere $S^{3}$ (i.e., $L=L_{1} \cup L_{2}$ where $L_{1}, L_{2}$ are disjoint polygonal simple closed curves in $S^{3}$. In Theorem 1 of this paper we give a geometric condition on $L$ which implies it is unknottable. In Theorem 2, we show that there is an infinite class of links of two components which are unknottable.
\end{abstract}

We call a continuous (PL) map $f: S^{3} \rightarrow S^{3}$ strongly $1-1$ on $L$ if $f \mid L$ is a homeomorphism onto $f(L), f\left(S^{3}-L\right) \cap f(L)=\varnothing$ and $f$ is locally $1-1$ at each point of $L$. In Theorem 1 of [3], the link $L_{0}=L_{01} \cup L_{02}$ where $L_{01}$ is unknotted and $L_{02}$ is the square knot is shown to have the property that there is no strongly $1-1$ map $f$ on $L_{0}$ such that $f\left(L_{01}\right)$ and $f\left(L_{02}\right)$ are unknotted. Call $L$ "unknottable" if there does not exist a strongly $1-1$ map $f$ on $L$ such that $f\left(L_{1}\right)$ and $f\left(L_{2}\right)$ are unknotted. This paper and [3] resulted from an attempt to generalize Hempel's result [2] that given any knot $K$ in $S^{3}$ there exists a strongly $1-1$ map $f$ on $K$ such that $f(K)$ is unknotted.

Let $S_{1}$ be a (PL) orientable surface such that $\mathrm{Bd} S_{1}=L_{1}$ and $L_{2}$ intersects and pierces $S_{1}$ in a finite number of points. Let $N(L)=$ $N\left(L_{1}\right) \cup N\left(L_{2}\right)$ be a regular neighborhood of $L$ such that $S_{1} \cap N\left(L_{1}\right)$ is an annulus and $S_{1} \cap N\left(L_{2}\right)$ consists of transverse disks. Call $S_{1}$ essential if $S_{1}-$ Int $N(L)$ is incompressible [7] and boundary incompressible [7] in $S^{3}-\operatorname{Int} N(L)$.

Definition $1 . \quad L$ is boundary incompressibly unlinked with respect to $L_{1}$ (B.I.U.) if, whenever $S_{1}$ is essential, we have $S_{1} \cap L_{2}=$ $\varnothing$. $L$ is said to be 1-linked [5] if $L_{1}, L_{2}$ do not bound disjoint orientable surfaces in $S^{3}$.

Theorem 1. If $L$ is 1-linked, B.I.U. and $L_{1}$ is knotted, then $L$ is unknottable.

Proof. Suppose there exists a $f: S^{3} \rightarrow S^{3}$ which is strongly $1-1$ on $L$ and $f\left(L_{1}\right), f\left(L_{2}\right)$ are unknotted. Let $D_{1}$ be a disk in $S^{3}$ such that Bd $D_{1}=f\left(L_{1}\right)$ and $f\left(L_{2}\right)$ intersects and pierces $D_{1}$ in a finite number $t$ of points. Suppose also that $t$ is chosen to be smallest possible. Now, following the techniques used in [7], we adjust $f$ so that it is transverse to $D_{1}$, in particular $D_{1}^{\prime}=f^{-1}\left(D_{1}\right) \cap\left(S^{3}-\operatorname{Int} N(L)\right)$ is an 
orientable surface with one boundary component in $\operatorname{Bd} N\left(L_{1}\right)$ which is a longitude of $N\left(L_{1}\right)$ and $t$ boundary components in $\mathrm{Bd} N\left(L_{2}\right)$, each of which is a meridian of $N\left(L_{2}\right)$. Now suppose $D_{1}^{\prime}$ is compressible in $S^{3}-\operatorname{Int} N(L)$, i.e. there exists a disk $Q$ in $S^{3}-\operatorname{Int} N(L)$ such that $Q \cap D_{1}^{\prime}=\mathrm{Bd} Q \cap D_{1}^{\prime}=\mathrm{Bd} Q$ and $\mathrm{Bd} Q$ does not bound a disk in $D_{1}^{\prime}$. Now if the loop $f(\operatorname{Bd} Q)$ separates a point of $D_{1} \cap f\left(L_{2}\right)$ from Bd $D_{1}$, we may apply Dehn's lemma [4] to conclude that $t$ was not minimal. If $f(\mathrm{Bd} Q)$ separates no point of $D_{1} \cap f\left(L_{2}\right)$ from $\mathrm{Bd} D_{1}$, then we may cut out a small regular neighborhood of $\mathrm{Bd} Q$ in $D_{1}^{\prime}$ and add two parallel copies of $Q$ to form a new orientable surface $D_{1}^{\prime \prime}$ with less genus than $D_{1}^{\prime}$. We may then redefine the map $f$ so that $D_{1}^{\prime \prime}=f^{-1}\left(D_{1}\right) \cap\left(S^{3}-\right.$ Int $\left.N(L)\right)$. If $D_{1}^{\prime}$ is boundary compressible, then there exists a disk $Q$ such that Int $Q \cap D_{1}^{\prime}=\varnothing$ and $\operatorname{Bd} Q$ consists of two arcs, one in $\mathrm{Bd} N\left(L_{2}\right)$, the other in $D_{1}^{\prime}$ and the arc in $D_{1}^{\prime}$ together with any arc in $\mathrm{Bd} D_{1}^{\prime}$ do not bound a disk in $D_{1}^{\prime}$. In this case we may use a modified version of the loop theorem (see [6]) on the loop $f(\mathrm{Bd} Q)$ in $S^{3}-$ Int $f(N(L))$ to conclude that $t$ was not minimal. Hence we may assume that $D_{1}^{\prime}$ is incompressible and boundary incompressible. Since $L$ is B.I.U. we have $t=0$. Then $f\left(L_{2}\right)$ bounds a disk $D_{2}$ which is disjoint from $D_{1}$. We may adjust $f$ so that $f^{-1}\left(D_{1}\right), f^{-1}\left(D_{2}\right)$ are disjoint orientable surfaces, contradicting the assumption that $L$ is 1 -linked, and the proof is complete.

We now define the class of links $L_{1 j} \cup L_{2 j}$ illustrated in Figures 1 and 2. Each $L_{1 j}$ is a curve with $j$ full twists ( $j$ is any positive or negative integer and one of the full twists is shown in the figure). If $j \neq 0$, then in [1] it is shown that $L_{1 j}$ is knotted.

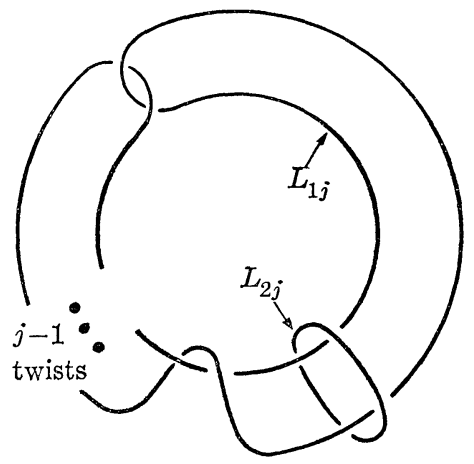

FIGURE 1

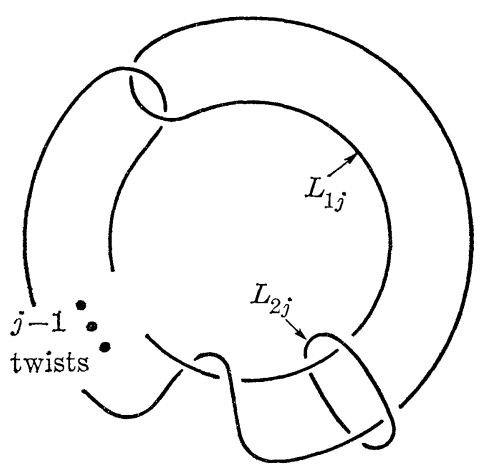

FIgURE 2

LEMMA 1. $L_{1 j} \cup L_{2 j}$ is 1-linked for all $j$.

Proof. Suppose $L_{1 j}, L_{2 j}$ bound disjoint orientable surfaces $S_{1 j}$, $S_{2 j}$, respectively. Let $D^{\prime}$ be a disk bounded by $L_{2 j}$ such that $L_{1 j}$ intersects and pierces $D^{\prime}$ in two points and the two components of 
$L_{1 j}-D^{\prime}$ self link each other. By cut and paste techniques (see [7] or we used some of these methods in Theorem 1) we may assume that (Int $\left.D^{\prime}\right) \cap S_{2 j}=\varnothing$ and $D^{\prime} \cap S_{1 j}$ consists of one arc connecting the two points of $D^{\prime} \cap L_{1 j}$. Let $D^{\prime \prime}$ be a disk whose boundary consists of the $\operatorname{arc} D^{\prime} \cap S_{1 j}$ and one of the two ares of $L_{1 j}-D^{\prime}$. Assume $D^{\prime \prime} \cap D^{\prime}=D^{\prime} \cap S_{1 j}$ and the other arc of $L_{1 j}-D^{\prime}$ intersects and pierces $D^{\prime \prime}$ in one point. But it now follows that there is a curve in $S_{2 j} \cap D^{\prime \prime}$ which is not homologous to zero in $S^{3}-L_{1 j}$, contradicting that $S_{1 j} \cap$ $S_{2 j}=\varnothing$.

In Figure 3 we view $L_{1 j}$ as being contained in a cube with two handles $C$ where $N\left(L_{1 j}\right) \subset \operatorname{Int} C \subset S^{3}-L_{2 j}$. Let $H_{1}, H_{2}$ be the two annuli illustrated in Figure 3, where $H_{1} \cap H_{2}$ is an arc.

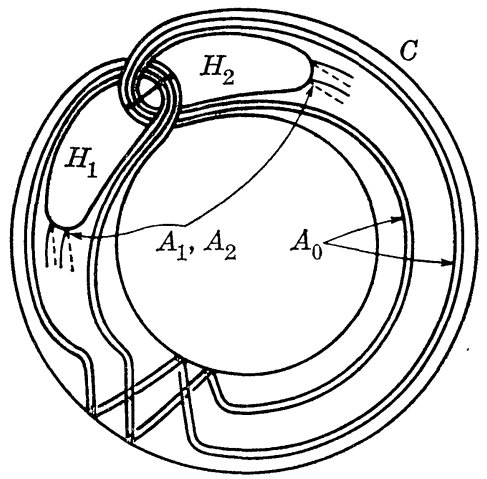

FIgURE 3

Lemma 2. Each link $L_{1 j} \cup L_{2 j}, j \neq 0$, is boundary incompressibly unlinked (B.I.U.).

Proof. Suppose $S^{\prime}$ is an orientable surface in the solid torus $T=S^{3}-$ Int $N\left(L_{2 j}\right)$ with one boundary component $L_{1 j}$ and each of the remaining $t$ boundary components is a meridian of $N\left(L_{2 j}\right)$ in Bd $N\left(L_{2 j}\right)$. Suppose also that $S=S^{\prime}-\operatorname{Int} N\left(L_{1 j}\right)$ is incompressible and boundary incompressible in $T-\operatorname{Int} N\left(L_{1 j}\right)$. We may choose the cube with two handles $C$ so that $S^{\prime} \cap C$ consists of an annulus $A_{0}$ and $s$ disks $A_{1}, \cdots, A_{s}$ (see Figure 3 ). Now, by following the techniques used in Lemma 1 of [3], we may adjust $S^{\prime}$ so that $S^{\prime} \cap H_{1}$ is one arc parallel to $H_{1} \cap H_{2}$ in $H_{1}$. (To see this, put $S^{\prime}$ in general position relative to $H_{1}$ and push ares of $S^{\prime} \cap H_{1}$ with both endpoints in the same component of $\mathrm{Bd} H_{1}$ off $H_{1}$ and then off $C$, i.e. we reduce $s$ by 1 or 2 and hence we may suppose $s=0$.) By the same reasoning we may suppose further that $S^{\prime} \cap H_{2}$ consists of one arc parallel to $H_{1} \cap H_{2}$ in $H_{2}$. Let $N\left(H_{1}\right), N\left(H_{2}\right)$ be regular neighborhoods of $H_{1}, H_{2}$, resp., taken in $T$ - Int $C$. Let $T^{\prime}$ be the solid torus 
$C \cup N\left(H_{1}\right) \cup N\left(H_{2}\right)$. Then $T$ - Int $T^{\prime}$ is homeomorphic to the product space $\left(S^{1} \times S^{1}\right) \times I$. None of the three simple closed curves of $S^{\prime} \cap$ $\mathrm{Bd} T^{\prime}$ is homotopic to the $t$ curves of $S^{\prime} \cap \mathrm{Bd} T$. (Note that one component of $S^{\prime} \cap \mathrm{Bd} T^{\prime}$ bounds a disk in $\mathrm{Bd} T^{\prime}$ and the other two go once around the longitude of $T^{\prime}$ and $j$ times, $j \neq 0$, around the meridian of $T^{\prime}$.) Since $S$ is incompressible and boundary incompressible, it follows that $\pi_{1}\left(S \cap\left(T\right.\right.$ - Int $\left.\left.T^{\prime}\right)\right)$ injects into the abelian group $\pi_{1}\left(T-\operatorname{Int} T^{\prime}\right)$. Hence $S \cap\left(T-\operatorname{Int} T^{\prime}\right)$ consists of one disk and one annulus, so $t=0$ and the proof of Lemma 2 is finished.

Theorem 1, Lemma 1 and Lemma 2 now imply the following:

THEOREM 2. Each of the links $L_{1 j} \cup L_{2 j}, j \neq 0$, is unknottable, i.e. there does not exist a strongly $1-1$ map $f$ on $L_{1 j} \cup L_{2 j}$ such that $f\left(L_{1 j}\right)$ and $f\left(L_{2 j}\right)$ are unknotted.

\section{REFERENCES}

1. R. H. Bing and J. M. Martin, Cubes with knotted holes, Trans. Amer. Math. Soc., 155 (1971), 217-231.

2. J. Hempel, A surface in $S^{3}$ is tame if it can be deformed into each complementary domain, Trans. Amer. Math. Soc., 111 (1964), 273-287.

3. Howard Lambert, Unknotting links in $S^{3}$ by maps, to appear in Proc. Amer. Math. Soc.

4. C. D. Papakyriakopoulos, Dehn's lemma and the asphericity of knots, Ann. of Math., 66 (1957), 1-26.

5. N. Smythe, Boundary links, Topology Seminar, Wisconsin, 1965, Annals of Mathematics Studies, no. 60, Princeton, 1966, pp. 69-72.

6. J. Stallings, On the loop theorem, Ann. of Math., 72 (1960) 12-19.

7. F. Waldhausen, On irreducible 3-manifolds which are sufficiently large, Ann. of Math., (2) 87 (1968), 56-88.

Received October 8, 1975 and in revised form April 26, 1976.

UNIVERSITY OF IOWA 


\section{PACIFIC JOURNAL OF MATHEMATICS}

\section{EDITORS}

RICHARD ARENS (Managing Editor) University of California

Los Angeles, California 90024

R. A. Beaumont

University of Washington

Seattle, Washington 98105
J. DUGUNDJI

Department of Mathematics University of Southern Californı

Los Angeles, California 90007

D. Gilbarg and J. Milgram

Stanford University

Stanford, California 94305

\section{ASSOCIATE EDITORS}
E. F. BECKENBACH
B. H. NeumanN
F. WOLF
K. YosHIDA

\section{SUPPORTING INSTITUTIONS}

UNIVERSITY OF BRITISH COLUMBIA

CALIFORNIA INSTITUTE OF TECHNOLOGY

UNIVERSITY OF CALIFORNIA

MONTANA STATE UNIVERSITY

UNIVERSITY OF NEVADA

NEW MEXICO STATE UNIVERSITY

OREGON STATE UNIVERSITY

UNIVERSITY OF OREGON

OSAKA UNIVERSITY
UNIVERSITY OF SOUTHERN CALIFORNIA

STANFORD UNIVERSITY

UNIVERSITY OF TOKYO

UNIVERSITY OF UTAH

WASHINGTON STATE UNIVERSITY

UNIVERSITY OF WASHINGTON

* * * *

AMERICAN MATHEMATICAL SOCIETY

NAVAL WEAPONS CENTER 


\section{Pacific Journal of Mathematics \\ Vol. 65, No. $1 \quad$ September, 1976}

David Lee Armacost, Compactly cogenerated LCA groups ............. 1

Sun Man Chang, On continuous image averaging of probability measures ...... 13

J. Chidambaraswamy, Generalized Dedekind $\psi$-functions with respect to a

polynomial. II................................... 19

Freddy Delbaen, The Dunford-Pettis property for certain uniform algebras ..... 29

Robert Benjamin Feinberg, Faithful distributive modules over incidence

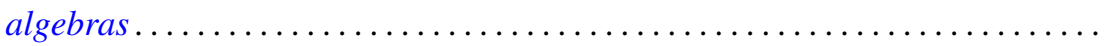

Paul Froeschl, Chained rings . . . . . . . . . . . . . . . . . . . .

John Brady Garnett and Anthony G. O'Farrell, Sobolev approximation by a sum

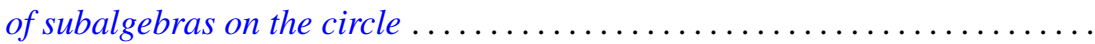

Hugh M. Hilden, José M. Montesinos and Thomas Lusk Thickstun, Closed

oriented 3-manifolds as 3-fold branched coverings of $S^{3}$ of special type.....

Atsushi Inoue, On a class of unbounded operator algebras ................

Peter Kleinschmidt, On facets with non-arbitrary shapes.

Narendrakumar Ramanlal Ladhawala, Absolute summability of Walsh-Fourier

series

Howard Wilson Lambert, Links which are unknottable by maps . . . . . . . . . . .

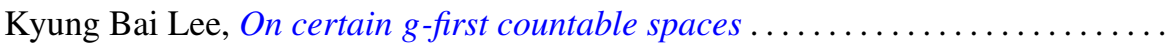

Richard Ira Loebl, A Hahn decomposition for linear maps .................

Moshe Marcus and Victor Julius Mizel, A characterization of functionals on $W_{1}^{p}$ possessing autonomous kernels. I . .

James Miller, Subordinating factor sequences and convex functions of several

variables.

Keith Pierce, Amalgamated sums of abelian l-groups ...

Jonathan Rosenberg, The $C^{*}$-algebras of some real and $p$-adic solvable

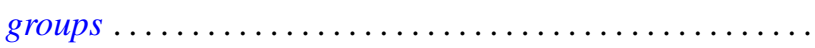

Hugo Rossi and Michele Vergne, Group representations on Hilbert spaces defined

in terms of $\partial_{b}$-cohomology on the Silov boundary of a Siegel domain . .

Mary Elizabeth Schaps, Nonsingular deformations of a determinantal

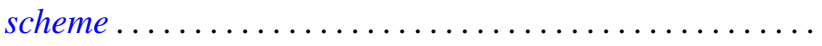

S. R. Singh, Some convergence properties of the Bubnov-Galerkin method...

Peggy Strait, Level crossing probabilities for a multi-parameter Brownian

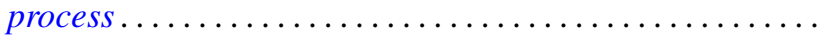

Robert M. Tardiff, Topologies for probabilistic metric spaces .

Benjamin Baxter Wells, Jr., Rearrangements of functions on the ring of integers of

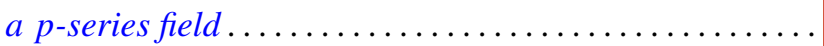

Robert Francis Wheeler, Well-behaved and totally bounded approximate identities for $C_{0}(X)$.

Delores Arletta Williams, Gauss sums and integral quadratic forms over local

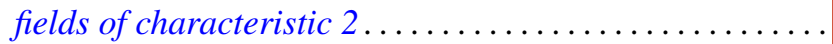

John Yuan, On the construction of one-parameter semigroups in topological 